\title{
CONFORMAL CAPACITIES AND EXTREMAL METRICS
}

\author{
JACQUELINE FERRAND
}

\begin{abstract}
For any non-compact Riemannian $n$-manifold $M$ it is proved that the function $\lambda_{M}^{\frac{1}{1-n}}: M \times M \rightarrow \overline{\mathbb{R}}_{+}$satisfies the triangular inequality. This result includes a Vuorinen conjecture. It follows from an extension to generalized condensers of a property of extremal functions.
\end{abstract}

\section{Introduction.}

For any non-compact Riemannian manifold $M$ of dimension $n \geq 2$ we previously defined a function $\lambda_{M}: M \times M \rightarrow \overline{\mathbb{R}}_{+}=\mathbb{R}_{+} \cup\{+\infty]$ only dependent on the conformal structure of $M$, and proved that for a class of manifolds containing all the proper subdomains of $\mathbb{R}^{n}, \lambda_{M}^{-\frac{1}{n}}$ was a distance on $M[\mathbf{F} 1, \mathbf{F} 2]$.

The case of a domain $G$ of $\mathbb{R}^{n}$ has been the object of several investigations leading to estimations of $\lambda_{G}\left[V_{1}, \ldots, V_{4}\right]$ and to properties of the $\lambda_{G}$-Lipschitz mappings $[\mathbf{F M V}]$. Then by considering the case of a ball M. Vuorinen has been led to conjecture that $\lambda_{G}^{\frac{1}{1-n}}$ is also a distance. In some way this improvement of my previous result is the best possible as it was proved in $[\mathbf{A V V}]$ that $\lambda_{G}^{-p}$ cannot be a metric if $p>\frac{1}{n-1}$. Moreover its interest is reinforced by the fact that, if $n=2$, it is relevant to the Teichmuller's theory of quadratic differentials and to the Jenkins's extremal metrics $[\mathbf{J} 1]$. This conjecture has been proven in $[\mathbf{A V V}]$ for $G=B^{n}$, by A.Y.Solynin $[\mathbf{S}]$ and J.A. Jenkins [J]2] for $G=\mathbb{R}^{2} \backslash\{0\}$, then extended by J.A. Jenkins [J2] to any plane domain with finite connectivity. But this extension is somewhat difficult to follow as it involves some families of homotopy groups which are not easy to define with precision when $G$ is not simply-connected.

In the present paper we will prove the following general result, which includes the Vuorinen conjecture:

Theorem A. With above notations, and for all $n \geq 2$, the function $m_{M}=$ $\lambda_{M}^{\frac{1}{1-n}}$ satisfies the triangular inequality. Consequently if $\lambda_{M}(x, y)$ is finite when $x \neq y$, the function $m_{M}$ is a distance on $M$.

We must emphasize that when applying this result to a domain $G$ of $\mathbb{R}^{n}$, we do not need any condition on the boundary of $G$, in opposition to Jenkins's theorem. 
JACQUELINE FERRAND

In fact this result will appear as a corollary of a general property of the level sets of extremal functions relevant to the theory of conformal capacities (Theorem B in Section 1).

This property is known in the case of classical condensers only $(\mathrm{cf}[\mathbf{H}]$ 3.8). We will easily extend it to the case of condensers associated with noncompact continua by dividing it in two reverse inequalities. Although the first inequality (2.1) is sufficient for proving Theorem A in Section 3, it will be useful to set the complete equality in view of other applications [F4]. Then we will interprete our results in terms of moduli (cf. Section 4).

At last in Section 5 we will prove the existence of extremal functions associated with a pair of points, and in some way the present study may be considered as an extension to Riemannian manifolds of Jenkins's theory of extremal metrics $[\mathbf{J} \mathbf{1}]$.

\section{Preliminaries.}

We will use the definitions and notations given in [F3]. Particularly $M$ will always denote a non-compact Riemannian manifold of dimension $n \geq 2$ and $\hat{M}=M \cup\{\infty\}$ its Alexandrov compactification.

A relative continuum of the manifold $M$ is a closed subset $C$ of $M$ without any compact connected component, or, in other terms: such that $C \cup\{\infty\}$ is connected in $\hat{M}$.

A function $u \in \mathcal{C}(M)$ is called monotone if for any relatively compact domain $D$ of $M$, the supremum and infimum of $u$ on $\partial D$ are respectively equal to its supremum and infimum on $D$.

We denote $H(M)=\mathcal{C}(M) \cap L_{n}^{1}(M)$ the space of real-valued continuous functions $u$ on $M$ admitting a generalized gradient $\nabla u$ with

$$
I(u, M)=\int_{M}|\nabla u|^{n} d \tau<+\infty,
$$

in which $d \tau$ is the volume element of $M$.

Let $H^{*}(M)$ denote the set of monotone functions $u \in H(M)$.

For any pair $\left(C_{0}, C_{1}\right)$ of closed subsets of $M$, the capacity of the condenser $\Gamma\left(C_{0}, C_{1}\right)$ is defined by

$$
\operatorname{Cap}\left(C_{0}, C_{1}\right)=\inf _{u \in A\left(C_{0}, C_{1}\right)} I(u, M)
$$

in which $A\left(C_{0}, C_{1}\right)$ is the set of those functions $u \in H(M)$, called admissible for $\Gamma\left(C_{0}, C_{1}\right)$, satisfying $u=0$ on $C_{0}, u=1$ on $C_{1}$ and $0 \leq u \leq 1$ everywhere. If $A\left(C_{0}, C_{1}\right)=\emptyset$ and, particularly, if $C_{0} \cap C_{1} \neq \emptyset$, we set $C a p\left(C_{0}, C_{1}\right)=+\infty$.

Our study will be based on the following previous result (cf. [F1] Th. 6.5 or [F3] Prop. 3.4): 
Theorem 1.1. Let $\left(C_{0}, C_{1}\right)$ be a pair of relative continua of $M$ such that $A\left(C_{0}, C_{1}\right) \neq \emptyset$. Then there exists a unique function $u \in A\left(C_{0}, C_{1}\right)$ satisfying $I(u, M)=\operatorname{Cap}\left(C_{0}, C_{1}\right)$.

This function, denoted extr $\left(C_{0}, C_{1}\right)$, is monotone on $M$, hence belongs to $H^{*}(M)$, and satisfies the variational condition

$$
\int_{M}|\nabla u|^{n-2} \nabla u . \nabla v d \tau=0
$$

for any function $v \in H(M)$ vanishing on $C_{0} \cup C_{1}$. In other terms, $u$ is $n$-harmonic on $M \backslash\left(C_{0} \cup C_{1}\right)$.

Remark. From ([F1] Prop. 4.2) or ([F3] Prop. 2.3) it follows that $u^{-1}(0)$ and $u^{-1}(1)$ are relative continua resp. containing $C_{0}, C_{1}$. It must be observed that, if $M \backslash\left(C_{0} \cup C_{1}\right)$ is not connected, $C_{0}$ [resp. $C_{1}$ ] can be a proper subset of $u^{-1}(0)$ [resp. $\left.u^{-1}(1)\right]$. In fact, if there exists a component $D$ of $M \backslash\left(C_{0} \cup C_{1}\right)$ such that $\partial D \subset C_{0}$ [resp. $\partial D \subset C_{1}$ ] we have necessarily $u=0$ [resp. $u=1$ ] on $D$. For that reason we will need the following lemma:

Lemma 1.3. Under the hypothesis of (1.1), the function $u=\operatorname{extr}\left(C_{0}, C_{1}\right)$ satisfies

$$
\operatorname{Cap}\left(u^{-1}(0), u^{-1}(1)\right)=\operatorname{Cap}\left(C_{0}, C_{1}\right) .
$$

Proof. Obviously $A\left(u^{-1}(0), u^{-1}(1)\right) \subset A\left(C_{0}, C_{1}\right)$, hence $C a p\left(u^{-1}(0), u^{-1}(1)\right)$ $\geq \operatorname{Cap}\left(C_{0}, C_{1}\right)$. The reverse inequality follows from the fact that $u$ is admissible for $\Gamma\left(u^{-1}(0), u^{-1}(1)\right)$, hence the announced result.

Now our main result can be stated as follows:

Theorem B. Let $C_{0}, C_{1}$ be two disjoint relative continua of a Riemannian manifold $M$ such that $\gamma=\operatorname{Cap}\left(C_{0}, C_{1}\right)<+\infty$, with $u=\operatorname{extr}\left(C_{0}, C_{1}\right)$, $n=\operatorname{dim}(M)$. Then for any pair $(\alpha, \beta)$ of real numbers with $0 \leq \alpha \leq \beta \leq 1$, the sets $C_{\alpha}^{-}=\{x \in M \mid u(x) \leq \alpha\}$ and $C_{\beta}^{+}=\{x \in M \mid u(x) \geq \beta\}$ are relative continua; the extremal function $u_{\alpha \beta}=\operatorname{extr}\left(C_{\alpha}^{-}, C_{\beta}^{+}\right)$is defined by $u_{\alpha \beta}=0$ on $C_{\alpha}^{-}, u_{\alpha \beta}=1$ on $C_{\beta}^{+}$and $u_{\alpha \beta}(x)=(u(x)-\alpha) /(\beta-\alpha)$ if $\alpha<u(x)<\beta$.

At last the capacity of $\Gamma\left(C_{\alpha}^{-}, C_{\beta}^{+}\right)$is $\gamma_{\alpha \beta}=\operatorname{Cap}\left(C_{\alpha}^{-}, C_{\beta}^{+}\right)=(\beta-\alpha)^{1-n} \gamma$.

The fact that $C_{\alpha}^{-}$and $C_{\beta}^{+}$are relative continua comes from ([F1] 4.2) or ([F3] 2.3). The other assertions will directly follow from the inequalities (2.1) and (4.2). In Section 3. we will obtain Theorem A as a corollary of the first inequality (2.1). 


\section{First inequality (majorization of $\gamma_{\alpha \beta}$ ).}

We observe that the variational equation (1.2) does not require any condition on $v$ at infinity. When $M$ is a domain $G$ of $\mathbb{R}^{n}$ we can interprete (1.2) by saying that $u$ is a weak solution of $\operatorname{div}\left(|\nabla u|^{n-2} \nabla u\right)=0$ on $G \backslash\left(C_{0} \cup C_{1}\right)$ satisfying $u=0$ on $C_{0}, u=1$ on $C_{1}$ and $\frac{d u}{d n}=0$ on $\partial G$.

Then by the same arguments as in $[\mathbf{H}]$ we obtain:

Proposition 2.1. With the notation of Theorem B we have:

$$
\operatorname{Cap}\left(C_{\alpha}^{-}, C_{\beta}^{+}\right) \leq(\beta-\alpha)^{1-n} \operatorname{Cap}\left(C_{0}, C_{1}\right)
$$

Proof. The function $u_{\alpha \beta}$ defined on $M$ by $u_{\alpha \beta}=0$ on $C_{\alpha}^{-}, u_{\alpha \beta}=1$ on $C_{\beta}^{+}$and $u_{\alpha \beta}=(u-\alpha) /(\beta-\alpha)$ on $M_{\alpha \beta}=M \backslash\left(C_{\alpha}^{-} \cup C_{\beta}^{+}\right)$is admissible for $\Gamma\left(C_{\alpha}^{-}, C_{\beta}^{+}\right)$ and also for $\Gamma\left(C_{0}, C_{1}\right)$. By using (1.2) with $v=u-u_{\alpha \beta}$ we get:

$$
\begin{aligned}
\operatorname{Cap}\left(C_{0}, C_{1}\right) & =I(u, M)=\int_{M}|\nabla u|^{n-2} \nabla u . \nabla u_{\alpha \beta} d \tau=(\beta-\alpha)^{n-1} I\left(u_{\alpha \beta}, M\right) \\
& \geq(\beta-\alpha)^{n-1} \operatorname{Cap}\left(C_{\alpha}^{-}, C_{\beta}^{+}\right)
\end{aligned}
$$

hence the announced result.

Now we can observe that $u_{\alpha \beta}$ is $n$-harmonic on $M_{\alpha \beta}$ and satisfies the same boundary conditions as $\operatorname{extr}\left(C_{\alpha}^{-}, C_{\beta}^{+}\right)$. Theorem B would immediately follow if the unicity of such a function would be proved. However, as we are here dealing with functions which are not in $W_{n, 0}^{1}$, it does not seem that this result can be considered as known for $n \geq 3$. We will achieve the proof in Section 4 by an elementary argument.

\section{Proof of Theorem A.}

We recall that the function $\lambda_{M}$ has been defined by

$$
\lambda_{M}(x, y)=\inf _{C_{x}, C_{y}} \operatorname{Cap}\left(C_{x}, C_{y}\right)
$$

where $C_{x}, C_{y}$ are relative continua resp. containing $x$ and $y$, hence $\lambda_{M}(x, x)=$ $+\infty$ for all $x \in M$.

As relative continua cannot reduce to single points, this function is strictly positive (cf. [F3] Prop. 3.6) but $\lambda_{M}(x, x)$ is not necessarily finite when $y \neq x$ : for example, as $H^{*}\left(E^{n}\right)$ is reduced to constant functions, $\lambda_{E^{n}}$ is identically $+\infty$ (cf. [F1] $\S 7$ ). We shall say that $M$ is of class $L$ if $\lambda_{M}(x, y)$ is always finite when $y \neq x$. For example, any proper subdomain of $E^{n}$ is of class $L$. 
More generally, an open submanifold $M$ of a compact manifold $N$ is of class $L$ if, and only if, $N \backslash M$ contains at least two points (cf. [F1] Th. 9.1). For a still more general result see ([F3], Prop.7.6).

Then Theorem A will come from the following result in which $M$ is not assumed to be of class $L$ :

Proposition 3.2. For any three points $x, y, z$ in $M$ such that $\lambda_{M}(x, y)<$ $+\infty$, the function $m_{M}=\lambda_{M}^{\frac{1}{1-n}}$ satisfies the triangular inequality

$$
m_{M}(x, y) \leq m_{M}(x, z)+m_{M}(z, y) .
$$

Proof. $\varepsilon>0$ given, let $C_{0}, C_{1}$ be two $r$-continua such that $x \in C_{0}, y \in C_{1}$ and $\operatorname{Cap}\left(C_{0}, C_{1}\right) \leq \lambda_{M}(x, y)+\varepsilon$. From (1.3) we are allowed to assume that $u=\operatorname{extr}\left(C_{0}, C_{1}\right)$ satisfies $u^{-1}(0)=C_{0}, u^{-1}(1)=C_{1}$. Then let us set $\alpha=u(z)$ :

If $\alpha=0, z \in C_{0}$ and $\lambda_{M}(z, y) \leq \operatorname{Cap}\left(C_{0}, C_{1}\right)$.

If $\alpha=1, z \in C_{1}$ and $\lambda_{M}(x, z) \leq \operatorname{Cap}\left(C_{0}, C_{1}\right)$.

If $0<\alpha<1$, with the notations used in Theorem B, $z \in C_{\alpha}^{-} \cap C_{\alpha}^{+}$, hence from (2.1):

$$
\begin{aligned}
& \lambda_{M}(x, z) \leq \operatorname{Cap}\left(C_{0}, C_{\alpha}^{+}\right) \leq \alpha^{1-n} \operatorname{Cap}\left(C_{0}, C_{1}\right) \\
& \lambda_{M}(z, y) \leq \operatorname{Cap}\left(C_{\alpha}^{-}, C_{1}\right) \leq(1-\alpha){ }^{1-n} \operatorname{Cap}\left(C_{0}, C_{1}\right) .
\end{aligned}
$$

By raising the first members of these inequalities to the negative power $1 /(1-n)$ and adding them, we obtain

$$
m_{M}(x, z)+m_{M}(z, y) \geq\left[\operatorname{Cap}\left(C_{0}, C_{1}\right)\right]^{\frac{1}{1-n}} \geq\left[\lambda_{M}(x, y)+\varepsilon\right]^{\frac{1}{1-n}}
$$

and the same inequality holds when $\alpha=0$ or $\alpha=1$.

Hence (3.3) when $\varepsilon$ tends to zero.

\section{End of the proof of Theorem B.}

With the notations used in the statement of Theorem B let us set:

$$
v_{1}=\operatorname{extr}\left(C_{0}, C_{\alpha}^{+}\right), v_{2}=\operatorname{extr}\left(C_{\alpha}^{-}, C_{\beta}^{+}\right), v_{3}=\operatorname{extr}\left(C_{\beta}^{-}, C_{1}\right) .
$$

For any positive numbers $t_{1}, t_{2}, t_{3}$ with $t_{1}+t_{2}+t_{3}=1$ the function $v=$ $\sum_{i=1}^{3} t_{i} v_{i}$ is admissible for $\Gamma\left(C_{0}, C_{1}\right)$.

As the open sets $M_{0 \alpha}, M_{\alpha \beta}$ and $M_{\beta 1}$ are mutually disjoint we have:

$$
\gamma=\operatorname{Cap}\left(C_{0}, C_{1}\right) \leq I(v, M)=\sum_{k=1}^{3} t_{k}^{n} I\left(v_{k}, M\right)
$$


hence by choosing $t_{1}=\alpha, t_{2}=\beta-\alpha, t_{3}=1-\beta$ :

$$
\gamma \leq \alpha^{n} I\left(v_{1}, M\right)+(\beta-\alpha)^{n} I\left(v_{2}, M\right)+(1-\beta)^{n} I\left(v_{3}, M\right) .
$$

Now by applying (2.1) three times we have:

$$
I\left(v_{1}, M\right) \leq \alpha^{1-n} \gamma, I\left(v_{2}, M\right) \leq(\beta-\alpha)^{1-n} \gamma, I\left(v_{3}, M\right) \leq(1-\beta)^{1-n} \gamma .
$$

The second member of (4.1) is therefore not greater than $\gamma$, and all the inequalities (4.2) are reduced to equalities. Particularly we have

$$
\operatorname{Cap}\left(C_{\alpha}^{-}, C_{\beta}^{+}\right)=I\left(v_{2}, M\right)=(\beta-\alpha)^{1-n} \gamma=I\left(u_{\alpha \beta}, M\right)
$$

in which $u_{\alpha \beta}$ is the function defined in the proof of (2.1). Then it is obvious that $u_{\alpha \beta}=\operatorname{extr}\left(C_{\alpha}^{-}, C_{\beta}^{+}\right)$which completes the proof of Theorem B.

Interpretation in terms of moduli. With any condenser $\Gamma\left(C_{0}, C_{1}\right)$ one associates the family $\Sigma\left(C_{0}, C_{1}\right)$ of hypersurfaces contained in $M$ and separating $C_{0}$ from $C_{1}$. Then a non-negative Borel function $f$ is called admissible for $\Sigma\left(C_{0}, C_{1}\right)$ if, for any $\sigma \in \Sigma\left(C_{0}, C_{1}\right), \int_{\sigma} f d H^{n-1} \geq 1$ where $d H^{n-1}$ is the $(n-1)$-dimensional Hausdorff measure.

The conformal modulus of $\Sigma\left(C_{0}, C_{1}\right)$ is defined by:

$$
\operatorname{Mod}\left(C_{0}, C_{1}\right)=\inf _{f} \int_{M} f^{m} d \tau \quad\left(m=\frac{n}{n-1}\right)
$$

where $f$ is admissible for $\Sigma\left(C_{0}, C_{1}\right)$.

The formula of duality

$$
\operatorname{Mod}\left(C_{0}, C_{1}\right)=\left(\operatorname{Cap}\left(C_{0}, C_{1}\right)\right)^{\frac{1}{1-n}}
$$

has been proved by Gehring [G1] for smooth separating surfaces, then by Ziemer $[\mathbf{Z}]$ for arbitrary sets by using the Fuglede's notation of modulus of a system of measures $[\mathbf{F u}]$. In those theories $M$ is a domain of $\mathbb{R}^{n}$ and $C_{0}$, $C_{1}$ are two compact sets contained in $\bar{M}$ (see also [C]). In order to avoid all difficulty for extending (4.4) to the present case, we can use the functional definition of admissible functions, not very far from Ziemer's one, given in $[$ BLF $]$ with suitable justifications:

Definition 4.5. Let $C_{0}, C_{1}$ be two relative continua of $M$. A nonnegative function $f$ is called admissible for $\Sigma\left(C_{0}, C_{1}\right)$ if it belongs to $L^{m}(M)$ with $m=n /(n-1)$ and satisfies $\int_{M} f|\nabla u| d \tau \geq 1$ for any $u \in A\left(C_{0}, C_{1}\right)$. 
With that definition it is easy to check that the infimum in (4.3) is obtained with $f=|\nabla u|^{n-1} / I(u, M)$ in which $u=\operatorname{extr}\left(C_{0}, C_{1}\right)$, hence (4.4).

Then we have in the most general case

$$
m_{M}(x, y)=\lambda_{M}^{\frac{1}{1-n}}(x, y)=\sup _{C_{x}, C_{y}} \operatorname{Mod}\left(C_{x}, C_{y}\right)
$$

where $C_{x}, C_{y}$ are relative continua resp. containing $x, y$.

The extremal distance $m_{M}$ is thus defined in terms of moduli and we can observe that the second part of the proof of Theorem B is similar to the argument used in ([M] 7.2) for proving the general inequality

$$
\operatorname{Mod}(D) \geq \sum_{k=1}^{m} \operatorname{Mod}\left(D_{k}\right)
$$

in which the $D_{k}$ are mutually disjoint shells separating the boundaries of the shell $D$. But we emphasize the inefficiency of (4.7) for proving the Vuorinen conjecture, as this inequality is not in the good direction.

\section{Appendix. Some more results relative to the function $\lambda_{M}$. Extremal functions relative to a pair of points.}

It is noteworthy that we have set Theorem A without using the existence of a pair $\left(\gamma_{x}, \gamma_{y}\right)$ of relative continua satisfying $\operatorname{Cap}\left(\gamma_{x}, \gamma_{y}\right)=\lambda_{M}(x, y)$. We recall that the Jenkins's proof for plane domains was based on the existence of such continua which, in this special case, are trajectories of a quadratic defferential $Q(z) d z^{2}$ with poles at $x, y$. The following general result is therefore of some interest:

Theorem 5.1. For any pair $(x, y)$ of points in $M$ such that $\lambda_{M}(x, y)<+\infty$ there exists at least a pair $\left(\gamma_{x}, \gamma_{y}\right)$ of relative continua resp. containing $x, y$ with $\operatorname{Cap}\left(\gamma_{x}, \gamma_{y}\right)=\lambda_{M}(x, y)$. Hence the function $u=\operatorname{extr}\left(\gamma_{x}, \gamma_{y}\right)$ belongs to $H^{*}(M)$ and satisfies $I(u, M)=\lambda_{M}(x, y)$.

Proof. From $[\mathbf{F} 1]$ it is known that

$$
\lambda_{M}(x, y)=\inf _{u \in \alpha(x, y)} I(u, M)
$$

in which $\alpha(x, y)$ is the set of functions $u \in H^{*}(M)$ satisfying $u(x)=0$, $u(y)=1$.

If $\lambda_{M}(x, y)<+\infty$ there exists a sequence $\left(u_{k}\right)$ in $\alpha(x, y)$ such that $\lambda_{M}(x, y)=\lim I\left(u_{k}, M\right)$.

From classical properties of $H^{*}(M)$ (cf. [F3] Prop. 1.6) there exists a $c$ convergent subsequence of $\left(u_{k}\right)$ whose limit $u$ belongs to $\alpha(x, y)$ and satisfies 
$I(u, M) \leq \lim I\left(u_{k}, M\right)=\lambda_{M}(x, y)$; hence necessarily $I(u, M)=\lambda_{M}(x, y)$. Then $\gamma_{x}=u^{-1}(0)$ and $\gamma_{y}=u^{-1}(1)$ satisfy the required condition.

Remark. The following example shows that such a pair $\left(\gamma_{x}, \gamma_{y}\right)$ is not necessarily unique: if $M=\mathbb{R}^{n} \backslash\{0\}, x$ and $y$ being symmetrical with respect to 0 , there are two pairs of relative continua satisfying the required condition, symmetrical with respect to 0 : in one pair $\left(\gamma_{x}, \gamma_{y}\right) \gamma_{x}$ joins $x$ to 0 and $\gamma_{y}$ joins $y$ to $\infty$; in the other one $\gamma_{x}$ joins $x$ to $\infty$ and $\gamma_{y}$ joins $y$ to 0 .

Alternative definitions for $\lambda_{M}$. It is easy to see that we do not change the value of $\lambda_{M}(x, y)$ if in (3.1) we restict $C_{x}$ and $C_{y}$ to be connected (or, in other terms, to be non-compact continua, see [F3] §5). However another definition of $\lambda_{G}$ has been used in [V3] for a domain $G$ of $\mathbb{R}^{n}$ : this definition restricts $C_{x}$ and $C_{y}$ to be paths with endpoints on $\partial G$.

This alternative definition is perhaps not equivalent to our definition in the case of arbitrary domains. However, for domains $G$ with $\partial G$ of class $C^{1}$ the two definitions agree as we now show.

Sketch of the proof. Suppose $\lambda_{G}(x, y)<\infty$ and $\varepsilon>0$ given. Let $C_{0}, C_{1}$ be non-compact continua such that $\operatorname{Cap}\left(C_{0}, C_{1}\right)<\lambda_{G}(x, y)+\varepsilon$. If $\partial G$ has no double point, $\bar{C}_{0}$ and $\bar{C}_{1}$ cannot have a common point $a$ in $\partial G$ : otherwise, as $\partial G$ is of class $C^{1}$, it is easy to prove that any function $u \in A\left(C_{0}, C_{1}\right)$ would satisfy an inequality of the type

$$
\int_{0}^{R} \frac{\omega^{n}(r) d r}{r} \leq C_{n} I(u, G)
$$

in which $\omega(r)$ is the oscillation of $u$ on $G \cap S(a, r)$ and $C_{n}$ a constant. Hence a contradiction with the fact that for $r$ sufficiently small $\omega(r)=1$.

Then we can construct two polygonal lines $\gamma_{0}=\left[x, x_{1}, \ldots, x_{h}\right]$ and $\gamma_{1}=$ $\left[y, y_{1}, \ldots, y_{k}\right]$ with $x_{h} \in \partial G, y_{k} \in \partial G, x_{h-1}$ and $y_{k-1}$ being sufficiently near $\partial G, u\left(x_{i}\right)(i=1,2, \ldots, h-1)$ and $1-u\left(y_{j}\right)(j=1,2, \ldots, k-1)$ being sufficiently small, in such a way that $\operatorname{Cap}\left(\gamma_{0}, \gamma_{1}\right) \leq \operatorname{Cap}\left(C_{0}, C_{1}\right)+\varepsilon \leq$ $\lambda_{G}(x, y)+2 \varepsilon$. The same result holds when $\partial G$ has double points, but the proof is slightly more elaborated since we have to distinguish the two sides of $\partial G$.

\section{References}

[AVV] G.D.Anderson, M.K. Vamanamurthy and M. Vuorinen, Special functions of quasiconformal theory, Exposition. Math., 7 (1989), 97-138.

[BLF] J.P. Bardet and J. Lelong-Ferrand, Relation entre le q-module et la p-capacité d'un condensateur riemannien, C.R. Acad. Sci. Paris, 277, Série A (1973), 835-838. 
[C] P. Caraman, Le p-module et la p-capacité du cylindre, C.R. Acad. Sci. Paris, 290, Série A (1980), 829-832.

[F1] J. Lelong-Ferrand, Invariants conformes globaux sur les variétés riemanniennes, J. of Differential Geometry, 8 (1973), 487-510.

[F2] Construction de métriques pour lesquelles les transformations quasi-conformes sont lipschitziennes, Symposia Math. Vol. XVIII, INDAM, Academic Press, London, (1976), 407-420.

[F3] J. Ferrand, Conformal capacities and conformally invariant functions on Riemannian manifolds, Summed up in C.R.Acad. Sci.Paris, 318, Série I (1994), 213-216.

[F4] Conformal properties of Riemannian manifolds with at least two ends, Geometrial Dedicata, 61 (1996), 103-120.

[FMV] , G.J. Martin and M. Vuorinen, Lipschitz conditions in conformally invariant metrics, J. Analyse Math., 56 (1991), 187-209.

[Fu] B. Fuglede, Extremal lenght and functional completion, Acta Math., 98 (1957), 171-219.

[G1] F.W. Gehring, Extremal lenght definitions for the conformal capacity of rings in space, Michigan Math. J., 9 (1962), 137-150.

[G2] , Rings and quasiconformal mappings in space, Trans. Amer. Math. Soc., 103 (1962), 353-393.

[GLM] S. Granlund, P. Lindqvist and O. Martio, Conformally invariant variational integrals, Trans. Amer. Math. Soc., 277 (1983), 43-73.

[H] I. Holopainen, Non-linear potential theory and quasiregular mappings on Riemannian manifolds, Ann. Acad. Sci. Fenn. A.I. Dissertationes, 74 (1990), 1-45.

[J1] J.A. Jenkins, On the existence of certain general extremal metrics, I Annals of Math., 66 (1957), 440-453. II Tohoku Math. J., 45 (1993), 249-257.

[J2] _ On metrics defined by modules, Pacific J. Math., 167 (1995), 289-292.

[M] G.D. Mostow, Quasi-conformal mappings in n-space, Inst. Hautes Etudes Sci. Publ. Math., 34 (1968), 53-104.

[S] A.Y. Solynin, Moduli of 2-connected domains and conformally invariant metrics (Russian), Zapiski Nauts Semin. P.O.M.I., 196 (1991), 122-131.

[V1] M. Vourinen, Conformal invariants and quasiregular mappings, J. Analyse Math., 45 (1985), 69-115.

[V2] _ On Teichmuller's modulus problem in $\mathbb{R}^{n}$, Math. Scand., 63 (1988), 315333.

[V3] Conformal Geometry and quasiregular mappings, Lecture Notes in Math., Vol. 1319, Springer-Verlag, Berlin, 1988.

[V4] Conformally invariant extremal problems and quasiconformal maps, Quart. J. Math. Oxford (2), 43 (1992), 501-514.

[Z] W.P. Ziemer, Extremal lenght and conformal capacity, Trans. Amer. Math. Soc., 126 (1967), 460-473.

Received April 5, 1994 and revised February 14, 1995.

Universite Pierre et Marie Curie

14 RUE DE BAGNEUX

F 92330 Sceaux, France 